\title{
Hacia una gerencia social eficiente en América Latina*
}

\section{Bernardo Kliksberg **}

\section{Carácter estratégico de la inversión social}

Una nueva visión de los impactos y beneficios del empleo eficiente de recursos en el campo social está en plena ebullición actualmente en el mundo. El Premio Nobel Amartya SEN señala que ha habido un profundo error conceptual. Se ha marginado, postergado, o sacrificado el gasto en lo social en nombre de consideraciones cortoplacistas. Según indica, la evidencia de fines del siglo XX demuestra que ese gasto es una palanca eje del desarrollo futuro. Educación, salud y nutrición, entre otros, no son en realidad agastos. sino inversiones. de muy alta rentabilidad, y los paises que han asignado recursos sostenidos a estos campos, y los han manejado con eficiencia, están cobrando ahora réditos muy importantes en términos de progreso, y ventajas competitivas ${ }^{1}$. Por su parte, el Presidente del Banco Mundial James WOLFENSOHN afirma que asin desarrollo social paralelo, no habrá desarrollo económico satisfactorio" ${ }^{2}$. La existencia de desarrollo social no sólo es deseable por sí mismo por sus múltiples implicancias en el perfil de la sociedad; sin ese "piso" los avances económicos carecen de sustentabilidad.

En documentos recientes del Banco Mundial se plantea que existen cuatro tipos de capital en una sociedad: los activos naturales integrados por la dotación de recursos geográficos originarios de la misma, los activos producidos por la acción humana desde infraestructura hasta medios financieros, el capital humano conformado por la población, y el capital social integrado por los valores, las instituciones, la capacidad de asociacionismo, el clima de confianza entre los actores sociales y las pautas culturales, entre otros aspectos. Se señala que el capital humano, y el social, deben revalorizarse. En el siglo XXI su peso será decisivo. La calidad de los recursos humanos de un país, y la inteligencia. de sus instituciones, definirán seriamente su posición relativa en los procesos de globalización.

Hay datos crecientes que verifican estos planteos. Así, la inversión en recursos humanos a través de la ampliación y mejoramiento de los niveles educacionales tiene tasas de retomo macro y microeconómicas de excepción. El siglo XXI será aconocimiento intensivo. Como destaca Lester ThuRow, el conocimiento será la única fuente sostenida de ventajas comparativas ${ }^{3}$. Conocimiento implica una base de apoyo sólida y creciente en educación. Según sus datos, las empresas que invierten en educación de sus integrantes tienen una rentabilidad por dicha inversión que duplica a la de aquellas que invierten en planta y equipo.

Múltiples fuentes han indicado que actualmente una de las inversiones más rentables del planeta es la inversión en educación de niñas. Añadir un año más de escolaridad a las niñas de América Latina, por ejemplo, puede reducir la mortalidad infantil en un 9 por mil en la región. Los efectos de aumentar el capital cognoscitivo de las niñas, después mujeres, sobre sus pautas de fecundidad, y su desempeño preparto y postparto, son de gran envergadura.

En realidad, la educación es, como ha resaltado Nancy BIRDSall, "una forma fundamental de acumulación de capital. ${ }^{4}$.

En la misma dirección, la inversión en mejoramiento y ampliación del capital social de un país, en perfeccionar sus insti- 
tuciones, en crear nuevas formas y espacios organizacionales aptos para dar mejores respuestas, en generar redes que conecten a sus actores, en desarrollar sus potencialidades culturales, tiene efectos multiplicadores sobre el desarrollo. Entre otros ejemplos, un caso ilustrativo de cómo instituciones bien orientadas pueden ayudar a generar confluencias en áreas claves, y ello impactar la economía, es el del Ministerio de Comercio Exterior e Industria del Japón. El mismo consideró la falta de modernización de la pequeña empresa como un obstáculo fundamental para el crecimiento. A través de políticas promotoras y en diálogo continuo con los sectores empresariales impulsó la creación de condiciones favorables al ahorro y la inversión de las pequeñas y medianas empresas. El peso de ese "crecimiento desde abajo de la economía fue altamente significativo para el creciniento total.

En el nuevo razonamiento que emerge en el mundo de la globalización, la revolución tecnológica permanente, y la competitividad, comienza a haber cambios importantes en categorías tradicionales. En lugar de "gasto social", se habla cada vez más de inversión socialn.

Esa inversión no es simplemente un paliativo, de objetivos esencialmente asistenciales. Se trata de poner en marcha a través de ella "palancas centrales" para el crecimiento y la competitividad en las nuevas condiciones. La "inversión social" no es un gasto en consumo, administrada con eficiencia "crea capital", humano y social, y esa acumulación es insustituible para un desarrollo sostenido.

Todos estos replanteos tienen profundas implicancias para el futuro de América Latina, hoy un continente de inmensas potencialidades, pero al mismo tiempo amplísimos déficit sociales. Se estima que casi el $50 \%$ de la población de toda la región está por debajo de la línea de pobreza, hay altos niveles de deserción escolar, repitencia, limitada calidad de la educación, una tasa de escolaridad de sólo 5,2 años por habitante, el 41\% de la población padece algún grado de desnutrición, y otras agudas carencias. Asimismo, una de las características más significativas de la situación es que la región se ha convertido en la más desigual del mundo entero. Pobreza e inequidad traban el desarrollo, afectan la gobernabilidad democrática y producen severos sufrimientos a la población. Entre otras consecuencias, como destaca BIRDSALL, alas tasas de crecimiento en América Latina no pueden ser de más del 3 o el $4 \%$, en tanto no se cuente con la participación y el aporte de la mitad de la población que está comprendida en los percentiles más bajos de ingresos " 5 .

Se hacen necesarias amplias inversiones sociales operadas con eficiencia. Alli aparece un tema crucial: ¿Cómo hacer gerencia de excelencia en el campo social? Esta pregunta es esencial para los administraciones públicas latinoamericanas de las que se espera un vigoroso rol frente a los graves problemas sociales de la región.
La experiencia internacional indica que se trata de una problemática de alta complejidad y especificidad. Que una forma casi segura de obtener resultados limitados es, según han evidenciado numerosos programas, subestimar las dificultades y particularidades de la acción gerencial en esta materia. Hace falta un enfoque de agerencia social. ¿En qué consiste? Indagaremos algunos de los principales problemas gerenciales en esta materia, y luego reseñaremos algunos contenidos de ese enfoque. En ambos casos este trabajo tiene objetivos acotados. No se propone tratar detalladamente el tema, sino presentar una agenda sumaria de algunos de los principales problemas que implica y llanar la atención sobre la necesidad de darle alta prioridad.

\section{La agenda de problemas de la gerencia social}

Gerencia social eficiente tiene que ver con optimizar el rendimiento de los esfuerzos del Estado y los actores sociales en el enfrentamiento de los grandes déficit sociales de la región, y el mejoramiento del funcionamiento y resultados de la inversión en capital humano y capital social.

Practicarla requiere ante todo pasar del enfoque prescriptivo usual en gerencia en otros campos a un enfoque esencialmente -heurístico. Ésta no es un área donde los problemas se pueden solucionar recurriendo a "recetas" disponibles, o a manuales que prescriben qué se debe hacer. Es un terreno denso, surcado de complejidades, donde se debe hacer un trabajo heurístico, explorar la realidad, prestar máxima atención a sus particularidades, tener muy en cuenta las experiencias comparadas, construir conocimiento a través del ensayo y error, reajustar continuamente los marcos de referencia en función de los hechos. Marcando el carácter muy particular del campo, resalta Dennis Rondinelu, luego de analizar una amplio número de proyectos de desarrollo movilizados por agencias internacionales que tuvieron limitados resultados, que uno de los factores actuantes fue que irrespectivamente de la planificación del proyecto o de la forma en que se efectuó el análisis técnico raras veces se hace la observación de que los problemas encontrados eran impredecibles. ${ }^{6}$. La impredecibilidad a que alude está indicando los niveles de complejidad que pueden esperarse en la gestión de progranas sociales de amplio alcance.

¿Cuáles son algunas de las singularidades de la gerencia de programas sociales que determinan una agenda particular de problemas de gestión, que exige a su vez respuestas adecuadas a la misma? 
La experiencia en la ejecución de programas de amplio alcance en campos como educación, salud, desarrollo rural, mejoramiento urbano, empleo, agua, etc., indica que tienden a presentar entre otras las siguientes características:

a. Los objetivos de los programas están destinados con frecuencia a cumplirse en el mediano y largo plazo. Se trata de metas múltiples, y en diversos casos heterogéneas. Por otra parte, hay metaobjetivos que van más allá de los objetivos fijados. Se espera actualmente que los programas contribuyan a crear condiciones de autosostenimiento, es decir, que la comunidad asistida experimente en el proceso un crecimiento significativo en sus propias capacidades para continuarlos. Este metaobjetivo condiciona y enmarca las metas usuales. En la realidad suelen darse programas que cumplen los objetivos operativos trazados, pero que, después de un periodo de haber finalizado la cooperación prestada, fracasan finalmente, porque no ha habido un aporte real a su autosustentabilidad.

b. Las variables contextuales que pueden incidir sobre el funcionamiento efectivo de los programas sociales comprenden un espectro muy amplio de naturaleza variada. Normalmente en los estudios de prefactibilidad y en la elaboración de diseños se presta especial atención a los factores económicos y financieros. Sin embargo, hay otros aspectos que van a decidir fuertemente la marcha de los programas que debieran considerarse y someterse a exploraciones detenidas. Tales entre otros: el medio ambiente político en el que les va a corresponder actuar, el perfil demográfico de la población a asistir, los patrones culturales prevalentes y su posible compatibilidad o incompatibilidad con las propuestas de acción que el programa entraña, la historia particular de la comunidad asistida, y sus experiencias anteriores en esta materia, y las capacidades de articulación y organización comunitaria preexistentes.

c. En la ejecución efectiva de programas sociales de amplitud intervienen múltiples actores. Puede haber varias organizaciones ministeriales del Gobiemo Central, regiones, municipios, empresas, organizaciones no gubernamentales, sectores de la sociedad civil, las comunidades asistidas. Los programas sociales son, por naturaleza, implícita o explícitamente interorganizacionales.

Hay interdependencias básicas entre los actores participantes. Si las interdependencias subyacentes se movilizan positivamente el programa avanzará hacia sus metas. Si se convierten en "enfrentamientos jurisdiccionales. 0 epugnas interburocráticas. el programa tendrá serias dificultades. Se trata de ver entonces cómo se gestionan "conjuntos de organizaciones" y sus interacciones.

d. El proceso real de operación de estos actores múltiples, en contextos donde inciden numerosas variables de diferente extracción, suele ser complejo y fluctuante. Con frecuencia no es transparente, tras la superficie se están desarrollando diversas "disputas subterráneas. y *arreglos organizacionales.

e. Los gerentes sociales tienen en esos marcos que alcanzar las metas organizacionales, en medio de presiones diversas de diferente indole. Gestionan en el marco de un "campo de fuerzas". Un estudio típico sobre la situación real de egerentes sociales en operaciones. relativo a un programa público de subsistencias rurales en México describe de este modo los hechos: «El implementador es el foco de demandas y expectativas frecuentemente conflictivas de este conjunto de actores. Dentro de su propia organización está obligado a satisfacer las expectativas amplias y de largo plazo de sus superiores y subordinados. Los jefes de nivel nacional o regional, por ejemplo, hacen generalmente dos clases de demandas al implementador. Por una parte, esperan que use los recursos puestos a su disposición para alcanzar las metas y objetivos de los programas que han sido diseñados. Al mismo tiempo, esperan igualmente que el implementador mantenga una jurisdicción relativamente libre de conflictos abiertos en las áreas afectadas por la organización. Estas dos expectativas pueden proveer pautas-guías divergentes acerca de cómo asignar mejor los recursos públicos... Fuera del contexto organizacional el implementador es el foco de una variedad de demandas específicas de grupos o individuos" ${ }^{7}$.

f. Alcanzar efectividad y metas de autosustentación en programas sociales requiere, de acuerdo a las evidencias disponibles, crear espacios favorables a la participación activa de la comunidad asistida. La necesidad de promover la participación aparece como una exigencia organizacional básica. ¿Cómo se trabaja en este campo? ¿Qué se puede aprender de las dificultades que se han encontrado en el camino hacia la movilización del potencial latente en la participación? ¿Cómo se favorece la articulación de la comunidad para participar? ¿En qué instrumentos técnicos puede apoyarse una gestión facilitadora de la participación?

g. El monitoreo y la evaluación en la ejecución de programas sociales requieren un abordaje técnico particular, que permita que aporten elementos consistentes, y al mismo tiempo actúen como una herramienta de alta gerencia, y no como una simple rendición ex-post. Medir grado de cumplimiento de impactos, y resultados, en 
este campo necesita de metodologías adecuadas que, junto a las dimensiones cuantitativas, den cuenta de aspectos cualitativos. Al mismo tiempo, los sistemas de medición deben estar funcionando en •tiempo real", para que sus productos puedan ser incorporados a la toma de decisiones gerencial. Esa incorporación debe asegurarse organizacionalmente estableciendo mecanismos apropiados de procesamiento y análisis de los productos del monitoreo y la evaluación.

h. Los programas sociales deben dar cuenta permanente sobre la marcha y resultados de su gestión. La itransparencia debe ser activa" y debe garantizar la escrupulosidad de la operación. ¿Qué tipo de procesos y controles pueden cumplir estos objetivos?

i. Como sugería RonDinew, la operación simultánea de todas estas variables da lugar a programas que con frecuencia presentan escenarios no solamente ajenos a los planificados, sino dificiles de prever de antemano. Los programas se ejecutan con frecuencia en medio de condiciones de turbulencia. Cambian sobre la marcha datos claves, bajo la presión de variables externas, y modificaciones en la misma comunidad.

Ello plantea requerimientos muy importantes en términos de capacidad de respuesta y flexibilidad. ¿Se necesita un estilo gerencial acorde a ellos? ¿Cómo puede configurarse?

Gerencia social significa en la práctica cotidiana enfrentar -singularidades. como, entre otras, las enunciadas. No es una operación organizacional ‘tubular, sino otra de índole diferente, mucho más fluida, y de composición interorganizacional.

¿Qué enseña la experiencia comparada? ¿Cómo han logrado efectividad los programas sociales exitosos? A continuación se indaga ese terreno.

\section{Direcciones de trabajo avanzadas en gerencia social}

Las "singularidades" que se presentan en la ejecución de programas sociales masivos plantean un cuadro de dilemas gerenciales de carácter muy específico. Su abordaje necesita de un -enfoque de gerencia social", que parta de dichos dilemas. Ese enfoque es, como ya se anticipó, de carácter heurístico. Se basa en explorar toda esta área desde los marcos de referencia de las nuevas fronteras tecnológicas en gerencia de organizacio- nes, buscando al mismo tiempo aprender de los emensajes" que van dejando las experiencias concretas.

Algunas de las direcciones de trabajo más dinámicas en avance en gerencia social son las que se indican sintéticamente a' continuación:

\section{A. Desarrollo de un estilo gerencial uadaptativo»}

Las características de los programas sociales con un alto grado de "variedad. en las situaciones emergentes, y con márgenes significativos de impredecibilidad, exigen un estilo gerencial que estreche el contacto con la realidad, capte las señales. de la misma, practique las transformaciones necesarias en las programaciones previas, y responda en el timing acelerado que se requiere. Ese estilo implica un cambio de fondo en la tradicional separación entre planeación y acción. En la gerencia adaptativa ambos momentos se acercan al máximo posible. Se prevé, actúa, retroalimenta la previsión sobre la marcha, y actúa nuevamente, en casi una unidad fusionada.

Ese estilo requiere de gerentes que abajen. al terreno. Es incompatible con las usuales "campanas de cristal. que se conforman en altos niveles de las organizaciones. La frecuente situación señalada por Henry MINTzBERG en gerencia actual •Muchos gerentes no saben qué pasa en el floor donde está la línea y los clientes. ${ }^{8}$, choca frontalmente con las necesidades de adaptabilidad gerencial propias de los programas sociales.

\section{B. Análisis sistemático del medio ambiente gerencial}

El contexto incidirá sobre los procesos de ejecución de los programas sociales. Si se analiza con herramientas adecuadas, será posible poner a foco oportunidades y riesgos que devienen del mismo. El análisis debe comprender dimensiones políicas, culturales y sociales y otras junto a las usuales de carácter económico y financiero. Hay instrumentos modernos que pueden hacer ricos aportes como la confección de mapas de actores, y el análisis de stakebolders.

\section{Reemplazo del enfoque sectorial por el abordaje integral}

La práctica de los programas sociales públicos ha sido con frecuencia en la región marcadamente sectorialista. Se intentan solucionar los problemas de deserción o baja calidad educativa por ejemplo desde el interior del sistema educativo. 0 temas como los déficit nutricionales, o la morbinatalidad, en el marco de los sistemas de salud. En la realidad cada una de las problemáticas sociales tiene raíces múltiples, y profundamente interre- 
lacionadas. Su superación necesita pasar de una visión sectorial a una comprensión más inteyral. Ello tiene implicancias directas en términos de acción. En iugar de "proteger" cuidadosamente las fronteras sectoriales, se hace imprescindible, por lo contrario para lograr mayor efectividad final, explorar las interconexiones y formular diseños de trabajo a partir de esa visión integral.

\section{Desarrollo de capacidades para la gerencia interorganizacional}

En los hechos en todo programa gerencial significativo participan diversos actores organizacionales. Su número se irá ampliando crecientemente en el futuro dados los avances en los procesos de descentralización del Estado y la integración cada vez más intensa de organizaciones de la sociedad civil. A su vez, el factor antes destacado, el avance hacia enfoques integrales pluralizará sustantivamente el ámbito de organizaciones participantes. ¿Cómo se optimizan esfuerzos de estas características? El tema ha sido planteado normalmente en la región, como un problema de coordinación y se ha tratado de darle salidas de rcoordinación formal. En la realidad excede totalmente a la mera coordinación. Se trata de hacer "gerencia sinérgica", de aprovechar las potencialidades de complementación, integración, y externalidades organizacionales" que pueden surgir de la acción conjunta de las organizaciones participantes. La coordinación lomal roza solamente la superficie de las sinergias posibles. Se detiene normalmente en un ámbito de intercambio restringido y no estratégico. En diversos casos, incluso, ese "como si se coordinaran. actúa como un sucedáneo de baja calidad de una acción sinérgica sustantiva y sostenida.

La búsqueda de vias para optimizar las sinergias plantea delicados problemas de compatibilización de autonomías organizacionales, políticas, y metodologías de acción de organizaciones con su propia historia y estilo. Las vías para abordarlos entrañan, entre otras tareas, la identificación de programas de trabajo conjunto, donde todos ganan, la negociación de términos de cooperación, y el establecimiento de mecanismos gerenciales de acción concertada, y de monitoreo y reajuste sobre la marcha. Los estilos gerenciales aplicables al manejo eficiente de esta gestión difieren marcadamente de los tradicionales. Entre otros, se habla en este campo de los modelos del "mediador. que trata de salvar las distancias entre las organizaciones, el "director de orquesta que procura actúen bajo reglas conjuntas, y el productor de cine que trata de integrar participativamente a las diversas organizaciones en un proyecto compartido.

El desenvolvimiento de capacidades para hacer gerencia sinérgica en lo social aparece como una de las oportunidades mayores para aumentar la eficiencia y el impacto de los programas sociales.

\section{E. Potenciación de la descentralización}

La descentralización aparece actualmente como una *vía regia para mejorar la gestión social. Implica acercamiento a los asistidos, más alta flexibilidad y agilidad de los actores y de los enfoques aplicados, posibilidad mayor de control de los programas por el conjunto social. Sin embargo, la experiencia está demostrando que no es un camino lineal. Descentralizar programas sociales con efectividad significa resolver una serie amplia de problemas. Entre ellos producir las transformaciones necesarias en los niveles que delegan facultades y recursos, y establecer puntos de coordinación operativos. También superar ciertos riesgos que se están presentando en la descentralización en la región. Si la descentralización se materializa aplicando a nivel regional o municipal el mismo tipo de modelos burocráticos, de carácter rígido utilizado a nivel central, sus efectos serán limitados, porque sus ventajas mayores desaparecerán. Un analista agudo de la gerencia moderna advierte sobre otro peligro. En opinión de Henry MINTZBERG, si descentralizar se sustancia en la imposición de metas cuantitativas fijas y rígidas a los niveles descentralizados, y en presionar para que se cumplan, esa dinámica lleva a un efecto .recentralizador. Respondiendo a la misma los directivos de las instancias descentralizadas preocupados por cumplir con las metas, centralizarán rígidamente hacia ellos mismos?

Potenciar las amplísimas posibilidades positivas que ofrece la descentralización significa poner a foco, y superar estos y otros riesgos, y crear condiciones favorables al aprovechamiento de sus ventajas comparativas.

\section{F. Modelos participativos}

La experiencia internacional reciente demuestra que una de las mejores posibilidades de mejorar la gestión social se halla en la movilización de slas promesas de la participación. en la formulación, implementación y evaluación de la misma. El tema ha atraído la atención de los principales organismos internacionales de financiamiento, y tanto el Banco Mundial como el BID han abierto amplias lineas de análisis, información y trabajo en derredor del mismo. Asimismo, aparece como un aspecto crucial en la propuesta de Desarrollo Humano presentada por todo el sistema de Naciones Unidas. Uno de los aspectos básicos de la revalorización de la participación en curso es la constatación de las estrechas correlaciones entre la meta de autosustentación de los programas sociales que orienta a la cooperación técnica internacional actualmente, y el grado de participación real de una comunidad asistida. El aumento de este orden de participación claramente contribuye a hacer crecer las posibilidades de autosustentación de los programas. 
Hay actualmente en participación una exploración intensa de experiencias fracasadas y exitosas, buscando claves que permitan abordajes más sólidos que en el pasado. Se están reestructurando activamente parte de los marcos conceptuales utilizados en esta área. Existe también una ampliación significativa del stock de técnicas aplicables a los procesos de participación.

La combinación de descentralización más participación aparece en el horizonte como un programa de acción en sí mismo para la gestión social. La descentralización con intervención creciente de la comunidad puede enfrentar algunos de los riesgos existentes en la misma, y desatar energías sociales latentes de gran consideración.

\section{G. Diseño de meta redes}

Las sociedades que han obtenido mejores resultados en gestión social han logrado avanzar en el camino de articular sistemáticamente las posibilidades de aporte de los diferentes actores, y crear redes y meta redes que los integren. En diversas sociedades avanzadas Estado, ONGs, sectores empresariales, laborales, iglesias, organizaciones de interés público no estatales, organizaciones voluntarias y comunidades asistidas, se hallan entrelazadas en redes que se plantean objetivos sociales mayores. En América Latina, es necesario pasar de la dispersión de esfuerzos al stejido. de estas redes. Ésta es una operación de ingeniería social. con impactos posibles de gran consideración. Las redes deberían a su vez ser operadas en base a criterios de gerencia interorganizacional moderna como los antes comentados. Entre otros casos, la operación de redes de este tipo, en campos como el mejoramiento de la calidad de la educación, parece haber sido una de las claves de los avances de los países de punta en esta área.

\section{H. Control social}

La gerencia social debe avanzar en la preparación e implantación de modelos que garanticen a la comunidad la plena transparencia y control sobre la marcha de los programas sociales. Al respecto es necesario avanzar en la construcción de indicadores precisos y claros de cumplimiento real de metas. Asimismo, renovar los mecanismos tradicionales de control. Una perspectiva promisoria es la que surge de las experiencias de "contraloría social., basadas en la participación activa de la comunidad asistida y la sociedad en general, en la evaluación continua de la labor de los programas sociales.

\section{Un desafío abierto: inversión social, desarrollo de la gerencia social y formación de gerentes sociales}

La capitalización en América Latina de las posibilidades existentes en las nuevas direcciones de trabajo en avance a nivel internacional en gestión de programas sociales requiere como condición necesaria de la preparación orgánica de gerentes sociales. La respuesta a las singularidades de la agenda gerencial que se presenta en este campo tiene que estar a cargo de recursos humanos especialmente calificados para afrontarlas. Esos gerentes deberán reunir un perfil de características particulares. Entre otros aspectos: tener orientación hacia el desarrollo de las capacidades de la comunidad, excelentes capacidades de concertación y negociación, aptitudes para la gestión interorganizacional, actitud de aprendizaje permanente de la realidad, manejo de las nuevas fronteras tecnológicas en gerencia, vocación de servicio firme y compromiso a fondo con los objetivos a lograr. Prepararlos requerirá un trabajo sistemático en el marco de una amplia red formativa a construirse. En su puesta en marcha pueden jugar un importante rol las organizaciones de la sociedad con mayor comprensión de la trascendencia de la variable gerencial.

Junto al avance en materia de sistemas formadores, será necesario progresar en la profesionalización de la gerencia social, y la conformación en este campo de una comunidad técnica que intercambie permanentemente experiencias y vaya estableciendo criterios orientadores. Al mismo tiempo, realizar una activa tarea de investigación y desarrollo en esta área que siga de cerca las experiencias nacionales, y la evolución internacional, y genere propuestas innovativas.

Hace falta montar una política orgánica de fortalecimiento y desarrollo de la gerencia social como campo de reflexión, estudio, formación y acción.

Corresponde hacer una puntualización. La gerencia por sí sola no solucionará los agudos problemas de pobreza y desigualdad de la región. Encararlos implica trabajar en múltiples planos. Uno de los más relevantes es desenvolver una amplia concertación social para lograr las profundas transformaciones necesarias. Pero los más imaginativos diseños de políica tendrán escasa concreción, si no se cuenta con administraciones públicas efi- 
cientes y una sólida gerencia social que garantice la aplicación de esos diseños ".

Nuevas ideas aparecen actualmente en el horizonte mundial sobre la temática social. La concepción de que la inversión social es imprescindible para avanzar un desarrollo sostenido tiene fuertes cimientos en la realidad histórica de nuestros días. Su práctica ha sido central en los éxitos de algunos de los países de mayor desarrollo reciente. América Latina atraviesa delicados problemas sociales. Como lo resalta el Banco Mundial, ellos son el :Talón de Aquiles para el desarrollo de la región. Insuficien- cias severas en educación, salud, nutrición y otras áreas críticas cruzan el Continente, que como marca dicha organización internacional aes notable como una región en que la pobreza, particularmente la pobreza absoluta, parece no registrar mejora alguna" ".

Probablemente uno de los mayores desafíos abiertos ante América Latina, a fin de este milenio, es movilizar para el enfrentamiento de esta problemática crucial una inversión social sostenida manejada con modelos de gerencia social avanzada. - Los puntos de vista expresados en este trabajo son los del autor y no reflejan nece-
sariamenie los de las inslituciones donde se desempena.

- Asesor de la ONU; OIT, UNESCO, UNICEF y otros. Autor de 33 libros y centenares de Imbajos de extendida utilización internacional. Rapponteur General del Congreso Inıcrnacional de Ciencias Adminisimaivas. Actualmente Coordinador del Instimsto Interamericano para el Desarrollo Social (INDES/BID, Washington). Entre sus obras se haIlan las siguientes editadas por el INAP de España, Gerencia Priblica en Tiempos de Incertidumbre, Parn inmestigar la Administración Piblica, La Refonma de la Administración Piblica en América Latina.

1 Amartya SI:N. Development thinking at the beginning of the XXI Century. Conferencia sobre .Development thinking and practice, BID, septiembre, 1996.

2 James Woulinsolın. El gasto social es clave. Clarin, Buenos Aires, 25 de febrero de 1996.

' Lester Tilukow, The Washington Post, 1996.

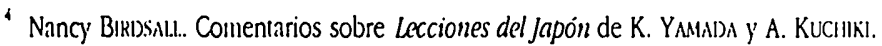
Conferencia sobre -Development thinking and practice. BID, septiembre, 1996.
3 !dem anterior. Los cuadros de pobreza e inequidad de América Latina son examinados sistemáticamente en Bernardo KLIKSBS:RG (comp.), Pobreza un tema impostergable, Fondo de Cultura Económica, 1997.

${ }^{6}$ Dennis A. RONI)Ni:ul. Development projects and policy experiments: an adaptative approacb 10 development administration. Methuen Editorial, New York, 1983.

7 Merilee S. GRINit)L:. El implementador: restricciones políicas sobre el desarrollo rural en México. Incluido en B. KLIKSBl:RG, J. Sulbranist (comps.), Para imiestigar la Administracion Priblica. Instiruto Nacional de Administración Pública de Espana, 1984.

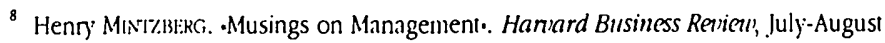
1996.

9 Idem anterior.

10 Sobre el papel de la administración pública en lo social puede verse: B. KLIKSBB:RC. -Repensando el rol del Estado en el Desarrollo Social. Más allá de dogmas y convencionalismos, Reforma y Democracia, Revista del CLAD, 1997.

"Shadid Bukki. Vicepresidente del Banco Mundial para América Latina. Agosto, 1996. 
